\title{
Semi-counterfactual Cryptography
}

\author{
Akshata Shenoy H. ${ }^{1}$, R. SRikanth ${ }^{2,3}$ and T. SRinivas ${ }^{1}$ \\ 1 ECE Dept, IISc, Bangalore \\ 2 Poornaprajna Institute of Scientific Research, Bangalore, India \\ ' 3 Raman Research Institute, Bangalore, India
}

PACS 03.67.Dd - Quantum cryptography and communication security

PACS 03.65. Ta - Foundations of quantum mechanics; measurement theory

PACS 03.67.Hk - Quantum communication

\begin{abstract}
In counterfactual quantum key distribution (QKD), two remote parties can securely share random polarization-encoded bits through the blocking rather than the transmission of particles. We propose a semi-counterfactual QKD, i.e., one where the secret bit is shared, and also encoded, based on the blocking or non-blocking of a particle. The scheme is thus semicounterfactual and not based on polarization encoding. As with other counterfactual schemes and the Goldenberg-Vaidman protocol, but unlike BB84, the encoding states are orthogonal and security arises ultimately from single-particle non-locality. Unlike any of them, however, the secret bit generated is maximally indeterminate until the joint action of Alice and Bob. We prove the general security of the protocol, and study the most general photon-number-preserving incoherent attack in detail.
\end{abstract}

Introduction. - Quantum key distribution (QKD) is a method allows two parties (Alice and Bob) to share ' a secret key, whose secrecy is protected by the laws of quantum mechanics (QM), such as no-cloning and the indistinguishability of non-orthogonal states [1]. It remains the most advanced application of quantum infor' mation theory experimentally [2, and even commercially. Since the proposal of the first QKD protocol [3], various paradigms of QKD have been proposed such as use of entanglement [4, 5, orthogonal states [6, 7, two-way communication [8 10, secure direct communication [11 14] and, most recently, counterfactual QKD (CQKD) [15, 16, which is based on the idea of interaction-free measurement 17. CQKD involves secret key sharing through blocking rather than transmission of a particle. The Noh protocol [15] has since been made more efficient [18] and its security investigated [19 21]. Recently, it was experimentally implemented [22].

In this work, we describe a scheme for CQKD which, unlike the counterfactual protocols of Refs. [15, 16], does not use polarization encoding. To see why this is interesting, it is worth noting that the idea of information transfer without particle transmission really means information transfer by blocking signals. This can be done classically, too. A 'classical' version of the Noh protocol would be: Alice transmits a red or blue ball to Bob, who randomly applies a color-based blocking operation. If he applies a red-blocker, and Alice transmitted a red ball, then he holds back the ball, else he transmits it back. Similarly, for the blue-blocker. Alice and Bob share a bit, determined by the ball color, whenever Alice does not receive a ball. Naturally, this classical protocol is completely insecure because Eve can determine the color of the ball during the forward transmission, and find out if Bob returns the ball. In the quantum mechanical version, however, Bob's blocking can spoil distructive interference on Alice's side, leading to a particle detection far away from Bob's blockade, which is the counterfactual element. If Eve tries to find the color (read: polarization), she commits the ball (read: particle) to the Alice or Bob path, potentially disrupting the path superposition state in which the particle would otherwise be prepared, which is the basis of security.

Now if both bits are to be generated by Bob's blocking act, then encoding on basis of some internal degree of freedom (such as polarization or spin) would be needed. If now we require counterfactuality only on one bit value, then one of the bits would be generated by blocking and the other by transmission. But in that case, the act of blocking or non-blocking itself could be used to encode bits, thereby 'freeing' polarization from the duty. Alice thus transmits a ball to Bob, and he blocks or does not block it. Alice deduces the bit according to whether she receives or does 
not receive his trasnmission. The second case represents the counterfactual bit. Our semi-counterfactual QKD protocol is the secure quantum version of this classical idea. Apart from the conceptual clarification it provides to the counterfactual paradigm in cryptography, it has the practical benefit of allowing polarization to be used to be used for security against certain Trojan horse attacks.

This article is structured as follows. In the following section, we present the new protocol, and compare and contrast it with some other related protocols in the subsequent section. Thereafter, the security of the protocol is discussed, followed by a discussion on the role of the translated no-cloning theorem in the present protocol. Next we prove the security under a general incoherent attack in the noiseless scenario. However, in practice, noise is ubiquitous. This is taken into consideration in the subsequent section, where we derive the tolerable error rate for a restricted, incoherent photon-number- preserving attacks.

A new scheme - - Light from the source $S$ hits the beamsplitter BS and splits into two beams one along Alice's arm $a(A)$ and another Bob's arm $b(A)$ respectively.The quantum state after BS is

$$
|\phi\rangle_{A B}=\sqrt{T}|00\rangle_{A}|\psi\rangle_{B}+i \sqrt{R}|\psi\rangle_{A}|00\rangle_{B},
$$

where $T$ and $R$ represent the coefficients of transmittance and reflectance of the BS respectively such that $T=1-R$, and $|00\rangle$ represents the vacuum state in the two polarization modes $H$ and $V$, while $|\psi\rangle$ represents a single photon state of arbitrary polarization, i.e., $|\psi\rangle=\alpha|10\rangle+\beta|01\rangle$ with $|\alpha|^{2}+|\beta|^{2}=1$. The first (second) ket refers to the transmitted (reflected) or Alice (Bob) arm.

Alice and Bob each possess a switch SW, an absorber Abs and a Faraday mirror FM. Each of the participants either applies the operation $F$ (reflect) or $A$ (absorb) depending on the state of the switch, which is randomly on or off, respectively. Ideally, the two SW's are independently controlled by a quantum random number generator. The three possibilties are: (1) Both SW's are on, so that Alice and Bob reflect their beams which causes an interference pattern at the detector $D_{1}\left(D_{2}\right)$ with probability $(T-R)^{2}$ $(4 R T)$; (2) One of the SW's is on while the other is off. This results in a detection at $D_{2}$ with probability $R T$, due to the physical travel of light along only one of the arms. If Alice (Bob) did the blocking, then the probability for detection at $D_{1}$ is $T^{2}\left(R^{2}\right)$, with the probability for absorption at the respective module being $R(T)$. This is the mode (on-off or off-on) in which a secret bit is generated. By convention, it is labelled either 0 or 1 depending on whether Alice or Bob applies the $A$ operation. The protocol is counterfactual with respect to Bob when secret bit 1 is generated; (3) The last possibility is that both the SW's are in the off mode resulting in Alice and Bob applying the operation $A$ and there is no detection both at $D_{1}$ and $D_{2}$.

The counterfactual nature of the protocol comes from the fact that the secret bit 1 is extracted by the

\begin{tabular}{c|c}
\hline Detection & Pattern (Alice, Bob) \\
\hline$D_{1}$ & $\left((F, A), \frac{1}{4}\right),\left((A, F), \frac{1}{4}\right)$ \\
\hline$D_{2}$ & $((F, F), 1),\left((F, A), \frac{1}{4}\right),\left((A, F), \frac{1}{4}\right)$ \\
\hline Null & $\left((F, A), \frac{1}{2}\right),\left((A, F), \frac{1}{2}\right),((A, A), 1)$ \\
\hline
\end{tabular}

Table 1: The allowed pattern of Alice's and Bob's action along with the probability that a detector click was produced.

interaction-free detection of Bob's $A$ setting. A detection at $D_{1}$ is determined by the presence of an absorber which did not scatter the particle. When secret bit 0 is generated, it is counterfactual with respect to the internal $\operatorname{arm} a$, but the photon travels physically along Bob's arm $b$. In this sense, the protocol is only semi-counterfactual with respect to the exposed arm.

The corresponding patterns of operations and outcomes are summarized in Table 1. The efficiency of the protocol is defined by the probability $P\left(D_{1}\right)=$ $P\left(D_{1} \mid(F, A)\right) P((F, A))+P\left(D_{1} \mid(A, F)\right) P((A, F))=\frac{1}{4} \frac{1}{4}+$ $\frac{1}{4} \frac{1}{4}=\frac{1}{8}$, which is the same as in the Noh protocol.

The protocol steps are as follows: (I) $n$ photons are sequentially injected from the left-hand side of the apparatus in Figure (11). (II) Depending on the random switch state, Alice applies $F$ or $A$ in the arm $a$ on each photon, and so does Bob in arm $b$. (III) On the $n$ outcome data collected, a fraction $f$ is randomly selected, for which Alice and Bob both announce their settings and outcome information. They check that the observed statistics is sufficiently close to Table 1. Two figures of merit are the visibility of the interference fringes

$$
\mathcal{V} \equiv \frac{P\left(D_{2} \mid F, F\right)-P\left(D_{1} \mid F, F\right)}{P\left(D_{1} \mid F, F\right)+P\left(D_{2} \mid F, F\right)} .
$$

and the error rate

$$
e \equiv P\left(F, F \mid D_{1}\right)+P\left(A, A \mid D_{1}\right),
$$

which estimates the fraction of mismatched secret bits. Two other figures of merit are estimates on $r$, the rate of multiple count, which may be due to dark counts or certain photon-number- non-preserving attacks, and $\lambda$, transmission loss rate over the channel. (IV) If $\mathcal{V}(e)$ is sufficiently close to $1(0)$, then the remaining approximately $(1-f) n / 8$ bits corresponding to $D_{1}$ detection are used for further classical post-processing to extract a smaller secure key via key reconciliation and privacy amplification.

If the timings of pulses where not random, and Eve knew them, she would probe Bob's setting by entering a photon into the path $b$ and studying it upon return, using an Alice-like set-up. As one method of protection against such a Trojan horse attack 21] Alice sends her pulses randomly. She and Bob later compare that in the events where Bob applied the operation $A$ and detected the photon, the time $t_{i}$ when the photon was sent by Alice satisfies $t_{s}=t_{r}-\tau$, where $t_{r}$ is the time of Bob's receipt, and $\tau$ the photon transit time. As a simpler alternative, Alice can vary the polarization randomly between the BB84 states 


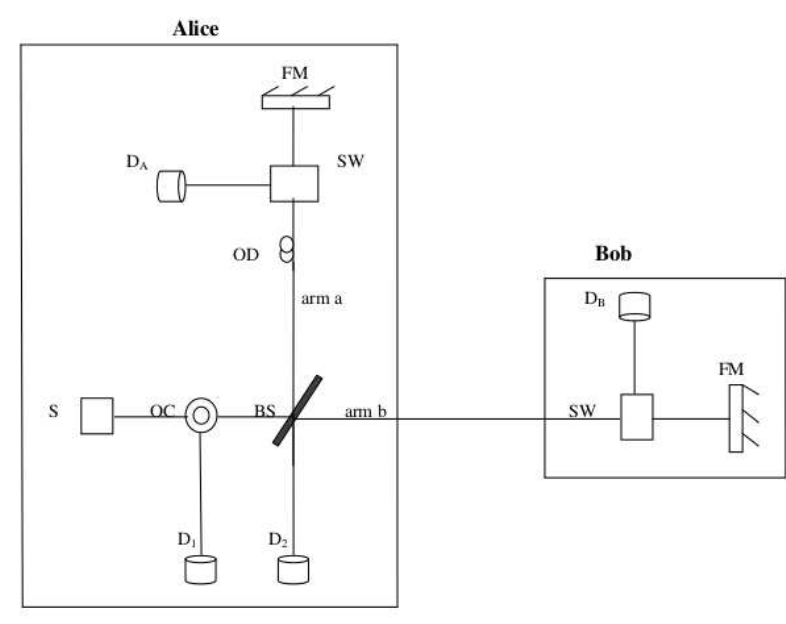

Fig. 1: Semi-counterfactual quantum cryptography: The experimental architecture of the proposed QKD using Michelsontype interferometer. Alice's station consisting of the source S initiates the protocol by sending light pulses through the optical circulator OC to the beamsplitter BS, which splits them into beams along arms $a$ and $b$. The optical delay OD maintains the phase between the arm by compensating for the path difference in the two arms. Light along arm a is subjected to absorption or reflection by Alice based on her switch state. Likewise by Bob along arm $b$ who also possesses SW, Abs and FM.

$|V\rangle,|H\rangle, \frac{1}{\sqrt{2}}(|V\rangle \pm|H\rangle)$ and Bob's $A$ operation may be accompanied by a polarization measurement. Trojan horse attacks by Eve to probe Bob's settings can be detected by later verifying the polarization in his detection events.

Comparison and contrast with some other protocols . - It is worth comparing and contrasting the present protocol with others that employ single photons: e.g., the counterfactual protocols, BB84 [3] or the Goldenberg-Vaidman protocol (GV) [6]. In all these cases, except BB84 (which uses conjugate coding), the encoding is with orthogonal states. In particular, the actions $(A, F)$ and $(F, A)$, when they can lead to a $D_{1}$ detection, produce the orthogonal states, $|00\rangle_{A}|\psi\rangle_{B}$ and $|\psi\rangle_{A}|00\rangle_{B}$, respectively. As with these other orthogonal-state-based protocols, single-particle non-locality, and restricting the observables that Eve can access, is the key to security.

Our protocol, however, differs from the other orthogonal-state-based protocols in one respect worth noting. The initial state transmitted by Alice is the same in all cases. Only after Alice's and Bob's actions is the secret bit generated. In GV, the secret bit is deterministic, while in the Noh protocol, Alice decides the polarization beforehand, so that if a detection is accepted (with probability $=1 / 8$ ), then the secret bit is fixed by the polarization chosen earlier. By contrast, in the present protocol, the secret bit is decided only after Alice's and Bob's actions.

Security . - Intuitively, security arises from singleparticle nonlocality: Eve's attempt to determine Bob's ac- tion will tend to localize the particle into one or the other arm, forcing a particle nature, and thereby disrupting the coherence between the two geographically separated wave packets in the two arms. This will be reflected in a reduction of visibility, which can be observed. However, the protocol is two-way, meaning that in some form (either physically or as a vacuum), the particle is re-exposed after Bob responds, and one needs to be sure that Eve cannot exploit the difference between the ingoing and outcoming states.

When the secret bit generated is 1, then Bob applied the operation $A$, and the photon never physically travelled on the exposed arm $b$. This seems to make the generation of this bit inherently safe from an eavesdropper Eve monitoring the arm. This is the intuition behind the expectation that counterfactuality helps security. However, quantum optically, non-travelling of a physical particle entails the travel of a vacuum pulse, with a non-trivial physical consequence. Thus a claim for security cannot be made without more detailed consideration.

Translated no-cloning theorem. In the Noh protocol, the reduced density operator of the particle in the exposed arm during the forward transmission is polarizationdependent, and thus correlated with the secret state. However, the correlated states are non-orthogonal, and the protocol's security is attributed to this non-orthogonality. In particular, Eve's action would be to evolve the exposed wave packet with an ancilla prepared in state $|m\rangle_{E}$ such that $U\left|s_{j}\right\rangle_{B}|m\rangle_{E}=\left|s_{j}\right\rangle_{B}\left|m_{j}\right\rangle_{E}$ with $j=1,2$. However, unitarity demands that $\left\langle s_{1} \mid s_{2}\right\rangle={ }_{B}\left\langle s_{1} \mid s_{2}\right\rangle_{B E}\left\langle m_{1} \mid m_{2}\right\rangle_{E}$, implying that for cloning to work, either $\left|s_{1}\right\rangle$ and $\left|s_{2}\right\rangle$ are orthogonal or $\left|m_{1}\right\rangle=\left|m_{2}\right\rangle$ and Eve gains no information. Thus Eve's any action that gains information will disturb the state of Alice's and Bob's particle, implying an information-vs-disturbance trade-off. In the backward transmission, both bit values are represented by the vacuum state.

In the present protocol, the state of the particle sent out by Alice is the same for either bit, namely $\frac{1}{2}|00\rangle\langle 00|+\frac{1}{4}(|01\rangle\langle 01|+| 10\rangle\langle 10|)$. But during the backward transmission, the states corresponding to the secret bits are represented in the exposed arm by $|00\rangle_{B}$ and $\frac{1}{2}\left({ }_{B}|01\rangle\left\langle\left. 01\right|_{B}+{ }_{B} \mid 10\right\rangle\left\langle\left. 10\right|_{B}\right)\right.$, which are orthogonal. The above reasoning would imply that our protocol is insecure. We will find below that this is not the case, mainly because the encoding states are not orthogonal to $|\phi\rangle_{A B}$, and that here too we have a situation with informationvs-disturbance trade-off.

General incoherent attack. In the most general, coherent attack, Eve prepares $n$ ancillas in the state $\left|\epsilon_{1}\right\rangle^{\otimes n}$ and another $n$ in the state $\left|\epsilon_{2}\right\rangle^{\otimes n}$. She has each particle interact with an ancilla prepared in state $\left|\epsilon_{1}\right\rangle$ using uni$\operatorname{tary} \mathcal{U}^{O}$ in the onward leg, and then with one prepared in state $\left|\epsilon_{2}\right\rangle$ using unitary $\mathcal{U}^{R}$ in the return leg. She waits until after Alice's announcement, to select a suitable basis to jointly measure all her ancillary pairs. 
We will focus on Eve's most general incoherent attack. Eve prepares an ancilla in state $|0\rangle$, and applies the operation (41) on the Bob-Eve system. We will assume some fixed polarization in this and the next subsection, and replace a double-ket with a single-ket to represent Alice's and Bob's particles, for simplicity.

$$
\begin{aligned}
|0\rangle_{B}|0\rangle_{E} & \rightarrow \alpha_{00}|0\rangle_{B}\left|\epsilon_{00}\right\rangle_{E}+\alpha_{0 \perp}\left|0^{\perp}\right\rangle_{B}\left|\epsilon_{0 \perp}\right\rangle_{E} \\
|1\rangle_{B}|0\rangle_{E} & \rightarrow \alpha_{10}|1\rangle_{B}\left|\epsilon_{10}\right\rangle_{E}+\alpha_{1 \perp}\left|1^{\perp}\right\rangle_{B}\left|\epsilon_{1 \perp}\right\rangle_{E}
\end{aligned}
$$

with $\left|\alpha_{00}\right|^{2}+\left|\alpha_{0 \perp}\right|^{2}=1$ and $\left|\alpha_{10}\right|^{2}+\left|\alpha_{1 \perp}\right|^{2}=1$, and $\left|0^{\perp}\right\rangle$ and $\left|1^{\perp}\right\rangle$ are states orthogonal to the vacuum state and $|1\rangle$, respectively. The fixed state transmitted over the channel and the ancilla evolves under (4) to

$$
\text { see eq. (5) }
$$

It suffices for our qualitative treatment to consider the state of the particle in the event when Alice and Bob apply the operation $F A$, and Bob registers a detection. It follows from Eq. (5) that the state of the particle is:

$$
\frac{1}{\sqrt{2}}\left(\alpha_{10}|0\rangle_{A}|0\rangle_{B}\left|\epsilon_{10}\right\rangle_{E}+\alpha_{0 \perp}|1\rangle_{A}\left(\hat{b}\left|0^{\perp}\right\rangle\right)_{B}\left|\epsilon_{0 \perp}\right\rangle_{E}\right),
$$

where $\hat{b}$ is the annihilation operator on Bob's mode, and we have conservatively set $\left|1^{\perp}\right\rangle \equiv|0\rangle$, implying that there is a probability $\left|\alpha_{0 \perp}\right|^{2}$ of a double or multiple count, which can be detected by Alice in principle.

We note that this detection occurs irrespective of whether Eve follows up with a further attack on the return leg, in particular, even if she 'unattacks', i.e., reverses the operation (4). The danger of producing multiple counts can be avoided by Eve if she uses a weaker attack that conserves photon number. We study this in the remainder of this section in a general setting.

General photon-number-preserving incoherent attack. The above general attack only indicates that the protocol is secure if no noise is detected. In practice noise will be unavoidable, even in the absence of an eavesdropper. On the other hand, conservatively, we must assume that all noise is due to Eve, and upper-bound her information on the secret key, from which the tolerable error rate can be derived. This task can be difficult for a general model of attack as above, and we adopt a simpler one here. On the onward leg, Eve prepares an ancilla in the state $|0\rangle_{E}$, and in the joint system $B E$, applies the number-preserving operation

$$
\mathcal{U}=|00\rangle_{B}\langle 00| \otimes U_{0}+\left(|01\rangle_{B}\langle 01|+| 10\rangle_{B}\langle 10|\right) \otimes U_{1},
$$

such that $\left\langle 0\left|U_{1}^{\dagger} U_{0}\right| 0\right\rangle \equiv\langle Y \mid N\rangle=\cos (\theta)$. Eve's attack in the onward leg followed produces the state:

$$
\mathcal{U}|\phi\rangle_{A B}|0\rangle=\frac{1}{\sqrt{2}}(|\psi\rangle|00\rangle|N\rangle+|00\rangle|\psi\rangle|Y\rangle) .
$$

The Alice-Bob action $(F, F)$ leaves the $|\phi\rangle_{A B}$ unchanged. In the case of $(F, A)$, the resulting states are $\frac{1}{\sqrt{2}}|00\rangle|00\rangle|Y\rangle$ or $\frac{1}{\sqrt{2}}|\psi\rangle|00\rangle|N\rangle$, of which the former implies detection by Bob and the latter leads potentially to a $D_{1}$ detection for secret bit 1 . In the case of $(A, F)$, the resulting states are $\frac{1}{\sqrt{2}}|00\rangle|00\rangle|N\rangle$ or $\frac{1}{\sqrt{2}}|00\rangle|\psi\rangle|Y\rangle$, of which the former implies detection by Alice and the latter leads potentially to a $D_{1}$ detection for secret bit 0 .

Suppose on the return leg, she 'unattacks' the same system, i.e., applies $\mathcal{U}^{\dagger}$, and measures the ancilla using a suitable positive operator-valued measure (POVM). After the "unattack", the case $F F$ results in $|\phi\rangle_{A B}|0\rangle$, implying null disturbance and also no information for Eve. In the case $(F, A)$, the resulting states are $\frac{1}{\sqrt{2}}|00\rangle|00\rangle|\tilde{Y}\rangle$ (where $\left.|\tilde{Y}\rangle \equiv U_{0}^{\dagger} U_{1}|0\rangle\right)$ or $|\psi\rangle|00\rangle|0\rangle$. In the case $(A, F)$, the resulting states are $\frac{1}{\sqrt{2}}|00\rangle|00\rangle|0\rangle$ or $\frac{1}{\sqrt{2}}|00\rangle|\psi\rangle|0\rangle$. Thus a detection of the probe is in the final state $|0\rangle$ whenever the particle is available for a $D_{1}$ detection,making Eve equally unable to say whether a 0 or 1 bit secret was generated. Allowing $\langle\tilde{Y} \mid 0\rangle=0$, Eve knows that Bob applied $A$, but these are precisely those events where Bob has a detection, making the particle unavailable for the secret-bit generation.

More generally, Eve may wish to use a general $\mathcal{U}^{\prime}$ rather than $\mathcal{U}^{\dagger}$ in the return leg. Then, if Alice and Bob applied $(F, F)$, the final state is $\frac{1}{\sqrt{2}}\left(|\psi\rangle|00\rangle\left|N^{\prime}\right\rangle+|00\rangle|\psi\rangle\left|Y^{\prime}\right\rangle\right.$, where $\left|N^{\prime}\right\rangle=U_{0}^{\prime} U_{0}|0\rangle$ and $\left|Y^{\prime}\right\rangle=U_{1}^{\prime} U_{1}|0\rangle$. In the case $(F, A)$, the resulting states are $\frac{1}{\sqrt{2}}|00\rangle|00\rangle\left|\tilde{Y}^{\prime}\right\rangle$ (where $\left.\left|\tilde{Y}^{\prime}\right\rangle \equiv U_{0}^{\prime} U_{1}|0\rangle\right)$ or $|\psi\rangle|00\rangle\left|N^{\prime}\right\rangle$. In the case $(A, F)$, the resulting states are $\frac{1}{\sqrt{2}}|00\rangle|00\rangle\left|N^{\prime}\right\rangle$ or $\frac{1}{\sqrt{2}}|00\rangle|\psi\rangle\left|Y^{\prime}\right\rangle$. In the cases of relevance, namely the cases $(F, F)$ and the subset of events leading to $D_{1}$ detection following applications of $(F, A)$ or $(A, F)$, the state of the probe is obtained by replacing $|N\rangle$ and $|Y\rangle$ with $\left|N^{\prime}\right\rangle$ and $\left|Y^{\prime}\right\rangle$ in relation to the situation where no attack takes place in the return leg.

Thus, the most general incoherent-number-preserving attack that Eve can launch would be to use the above onward leg attack, and then measure her probe $E$ after Alice's announcement. We will assume the worst-case scenario where Eve has complete knowledge of the transmission schedule between Alice and Bob. Thus she times her attack to happen just when the particle is about to enter Bob's station, and completes it after Alice's announcement of $D_{1}$ events.

It follows from the above that in such events, Eve would try to determine the secret bit by measuring her probe. She can optimally try to distinguish between $|N\rangle$ and $|Y\rangle$, which correspond to secret bits 0 and 1, respectively, using the following optimal POVM [23]:

$$
\begin{aligned}
M_{N} & =\frac{1}{1+|\langle N \mid Y\rangle|}(1-|Y\rangle\langle Y|), \\
M_{Y} & =\frac{1}{1+|\langle N \mid Y\rangle|}(1-|N\rangle\langle N|), \\
M_{\text {inconcl }} & =1-M_{N}-M_{Y},
\end{aligned}
$$

where outcome $M_{N}\left(M_{Y}\right)$ indicates deterministic outcome $|N\rangle(|Y\rangle)$ and $M_{\text {inconcl }}$ represents an inconclusive outcome. 


$$
\frac{1}{\sqrt{2}}(|0,1\rangle+|1,0\rangle)_{A B}|0\rangle_{E} \rightarrow \frac{1}{\sqrt{2}}\left(|0\rangle\left[\alpha_{10}|1\rangle\left|\epsilon_{10}\right\rangle+\alpha_{1 \perp}\left|1^{\perp}\right\rangle\left|\epsilon_{1 \perp}\right\rangle\right]+|1\rangle\left[\alpha_{00}|0\rangle\left|\epsilon_{00}\right\rangle+\alpha_{0 \perp}\left|0^{\perp}\right\rangle\left|\epsilon_{0 \perp}\right\rangle\right]\right)
$$

It follows from Eq. (10) that the probability of a conclusive read-out is $P_{c} \equiv 1-|\langle N \mid Y\rangle|=1-\cos (\theta)$.

Eve's information

$$
I_{B E}=P_{c}=1-\cos (\theta)=I_{A E} \equiv I_{E},
$$

the latter two equalities following from the symmetric nature of Eve's information after Alice's announcement. In the given direction of polarization of the photon, Alice's beam splitter may be represented as:

$$
\begin{aligned}
& d_{1}^{\dagger}=\frac{1}{\sqrt{2}}\left(a^{\dagger}+i b^{\dagger}\right) \\
& d_{2}^{\dagger}=\frac{1}{\sqrt{2}}\left(a^{\dagger}-i b^{\dagger}\right),
\end{aligned}
$$

where $a^{\dagger}, b^{\dagger}$ are the creation operators for the modes $A, B$, respectively, and $d_{1}$ and $d_{2}$ are annihilation operators corresponding to detections at $D_{1}$ and $D_{2}$, respectively. Hence, the state $|\phi\rangle_{A B}$ evolves to

$$
\left|\phi^{\prime}\right\rangle=\frac{1}{\sqrt{2}}\left(d_{1}^{\dagger}|0\rangle_{A B}|+\rangle_{E}+i d_{2}^{\dagger}|0\rangle_{A B}|-\rangle_{E}\right),
$$

where $| \pm\rangle_{E} \equiv \frac{1}{\sqrt{2}}\left(|N\rangle_{E} \pm|Y\rangle_{E}\right)$. From this, it follows that

$$
\begin{aligned}
\operatorname{Prob}\left(D_{2} \mid F F\right) & =\frac{1}{2}|\| N\rangle_{E}+|Y\rangle_{E} \|^{2} \\
& =\frac{1}{2}(1+\cos (\theta)) .
\end{aligned}
$$

We thus find that the visibility (2), conditioned on both applying $F$, falls from 1 to

$$
\mathcal{V}=\cos (\theta) \text {. }
$$

For any other of the three pairs of operations by Alice and Bob (e.g., $(A, F)$ ), an entanglement of the form (9) does not arise, and Eve's attack does not produce a deviation from the pattern in Table 1 .

Combining Eqs. (15) and (11), we find a complementarity relation

$$
\mathcal{V}+I_{E}=1,
$$

for the visibility during the $(F, F)$ instances and the information Eve gains during the $D_{1}$ outcomes. If the error rate is $e$, we have Bob's information to be $I_{A B}=1-h(e)$, where $h$ is the binary entropy. Assuming conservatively that there is no other noise than that due to Eve, and that she employs the above attack, the only changes to Table (11) is the addition of $\left(F, F, \frac{1}{2}(1-\cos (\theta))\right.$ in the $D_{1}$ row and modifying the $F F$ entry in the $D_{2}$ row to $\left(F F, \frac{1}{2}(1+\cos (\theta))\right.$. Thus error rate $e$ in Eq. (3) becomes, by Bayesian rule,

$$
\begin{aligned}
e & =P\left(F, F \mid D_{1}\right)=\frac{P\left(D_{1} \mid F, F\right) P(F, F)}{P\left(D_{1}\right)} \\
& =\frac{1-\cos (\theta)}{2-\cos (\theta)} .
\end{aligned}
$$

This is an estimate of the error in key, since $D_{1}$ events arising from an operation (F.F) leads to a mismatch in the private copies of the key. The condition for positive key rate in the protocol is 24]

$$
K=I_{A B}-\min \left\{I_{A E}, I_{B E}\right\}>0,
$$

where $K$ are the secret bits that can be distilled after Alice and Bob perform key reconciliation and privacy amplification. The condition for security in our protocol becomes, from Eqs. (16) and (18),

$$
h\left(\frac{1-\cos \theta}{2-\cos \theta}\right)<\cos \theta .
$$

or $\theta \lesssim 0.745 \mathrm{rad}$, which, in view of Eq. (3), implies $e \lesssim$ $20.9 \%$.

Discussion and conclusions . - We have presented a protocol for QKD which is counterfactual on one of the generated secret bits in that the encoding corresponds to the blocking or the non-blocking by Bob of a transmitted particle. This is motivated by observing that the information transfer via non-transmission of a particle in the return leg is possible even classically 25, as we noted earlier. Thus, this cannot be the basis for security. What this method does achieve is to make the information transfer counterfactual, whereby Alice is alerted to his blocking action by the photon detection at a detector where it would otherwise be absent (the fact that Bob's blockade influences the distant detector is the counterfactual element here [26]). By contrast, in a classically equivalent protocol, Alice would be alerted to his blocking by the non-return of the particle.

In the Noh protocol, Eve's attempt to eavesdrop the channel can lead to a blockade-like effect depending on the sharpness 27] of her eavesdropping operation, thereby potentially leading to Alice's counterfactual detection of the photon even when Bob does not block. This situation is not unlike in a conventional QKD protocol, such as BB84, where Eve disturbs the particle when her operation is inconsistent with Bob's. The fact that counterfactuality does not seem to offer any extra cryptographic advantage (though it gives a new form of communication in quantum cryptography) forms the basis of our departure from the Noh protocol.

Like the Noh, Pingpong, LM05 and Deng-Long 28] protocols, our scheme may be thought of as a two-way QKD protocol. In common with these, a practical implementation of our protocol faces certain difficulties because the photon has to travel twice the distance, which can limit range and key rate. Moreover, the two interferometric arms exist in different environments (an external fiber link 
to Bob and a spool at Alice's station), both requiring stabilization. We believe that these issues pose technological challenges, that can in principle be surmounted.

To protect the Noh protocol against Trojan-horse attack, one requires time-randomization of the sequence, for which the difficult task of rapid pulse referencing is required. With time randomization, the rate at which the key is generated is not constant, which is undesirable for network integration. By contrast, because we do not use polarization encoding, we have used it for detecting Trojan horse attacks, as discussed. Thus time randomization is not required and pulse referencing is less stringent in our protocol. Furthermore, because encoding is not polarization dependent, polarization drift poses a lesser problem in stabilizing interferometer.

Our protocol differs from each of the above two-way protocols in one or more key aspects. Our protocol has Alice sending a fixed state, unlike in the Noh and LM protocols. It involves single-particle nonlocality unlike in the LM protocol; finally, unlike in the Pingpong protocol, it does not involve two particle entanglement. A practical advantage of not using polarization encoding is that it makes the protocol secure against a kind of Trojan horse attack.

We have assumed zero transmission losses, so that every particle is accounted for by Alice's or Bob's detectors. Thus, a direction for generalizing our work is to allow for lossy channels. Another direction is to study how much a more general incoherent and even coherent attack, helps Eve.

\section{REFERENCES}

[1] Gisin N., Ribordy G., Tittel W. and Zisinden H., Rev. Mod. Phys., 74 (2002) 145.

[2] Scarani V., Bechmann-Pasquinucci H., Cerf N. J., Dušek M., Lütrenhaus N. and Peev M., Rev. Mod. Phys., 81 (2009) 1301.

[3] Bennett C. H. and Brassard G., Quantum cryptography: Public key distribution and coin tossing in proc. of Proc. IEEE Int. Conf. on Computers, Systems, and Signal Processing, Bangalore 1984 p. 175.

[4] Ekert A. K., Phys. Rev. Lett., 67 (1991) 661.

[5] Lo H.-K. and Chau H. F., Science, 283 (1999) 2050.

[6] Goldenberg L. and Vaidman L., Phys. Rev. Lett., 75 (1995) 1239.

[7] Avella A., Brida G., Degiovanni I. P., Genovese M., Gramegna M. and Traina P., Phys. Rev. A, 82 (2010) 062309.

[8] Boström K. and Felbinger T., Phys. Rev. Lett., 89 (2002) 187902.

[9] Lucamarini M. and Mancini S., Phys. Rev. Lett., 94 (2005) 140501.

[10] Sun M., Peng X., Shen Y. and H.Guo, Int. J. Quantum Inform., 10 (2012) 1250059.

[11] Deng F.-G. and Long G. L., Phys. Rev. A, 69 (2004) 052319 .

[12] Lu H., Fung C.-H. F., Ma X. and CAi Q.-Y., Phys. Rev. A, 84 (2011) 042344.
[13] Shukla C., Pathak A. and Srikanth R., Int. J. Quantum Inf., 10 (2012) 1241009.

[14] Eusebi A. and S.Mancini, Int. J. of Quant. Info., 9 (2011) 1209.

[15] Noh T.-G., Phys. Rev. Lett., 103 (2009) 230501.

[16] Salih H., Li Z.-H., Al-Amri M. and Zubairy M. S., Phys. Rev. Lett., 110 (2013) 170502.

[17] Elitzur A. C. and Vaidman L., Found. of Phys., 23 (1993) 987.

[18] Sun Y. and Wen Q.-Y., Phys. Rev. A, 82 (2010) 052318.

[19] Yin Z.-Q., Li H.-W., Chen W., Han Z.-F. and Guo G.-C., Phys. Rev. A, 82 (2010) 042335.

[20] Zhang S., Wang J., Jing Tang C. and Zhang Q., Chin. Phys. B, 21 (2012) 060303.

[21] Zhang S., Wang J. and TANG C. J., Europhys. Lett., 98 (2012) 30012.

[22] Brida G., Cavanna A., Degiovanni I. P., Genovese M. and Traina P., Laser Phys. Lett., 9 (2012) 247.

[23] Nielsen M. and Chuang I., Quantum Computation and Quantum Information (Cambridge) 2000.

[24] CsizÁr I. and Körner J., IEEE Trans. Inf. Theory, 24 (1978) 339.

[25] Gisin N., Optical communication without photons arXiv:1304.8053 (2013).

[26] Vaidman L., arXiv:1304.6689 (2013).

[27] Busch P. and Lahti P. J., Phys. Rev. D, 29 (1984) 1634.

[28] Deng F.-G. and Long G. L., Phys. Rev. A, 70 (2004) 012311. 